\title{
Comparison of bubble eruption models with two-fluid simulations in a 2D gas-fluidized bed
}

\author{
F. Hernández-Jiménez ${ }^{\mathrm{a}, *}$, J.R. Third ${ }^{\mathrm{b}}$, A. Acosta-Iborra ${ }^{\mathrm{a}}$, C.R. Müller ${ }^{\mathrm{b}}$ \\ a Universidad Carlos III de Madrid, Department of Thermal and Fluid Engineering. Av. de la Universidad 30, 28911 Leganés, Madrid, Spain \\ ${ }^{\mathrm{b}}$ ETH Zürich, Institute of Energy Technology, Laboratory of Energy Science and Engineering, Leonhardstrasse 27, 8092 Zurich, Switzerland
}

\begin{abstract}
A B S T R A C T
Two-fluid simulations of gas-solid fluidized beds are performed in this work with a threefold aim: (1) explore the capabilities of two-fluid modelling to reproduce realistically bubble eruption patterns in two-dimensional fluidized beds; (2) compare the results obtained from the two-fluid simulations with particle ejection models; and (3) provide information about the mutual interaction of the gas and particle flows during bubble eruption. To fulfil these aims, results from two-fluid simulations concerning the vertical and horizontal velocities of particles in bubble domes, prior to and during bubble eruption, are reported and compared with previously published experimental data taken from a bed of comparable geometry and operating conditions. The comparison shows excellent quantitative agreement. Particle ejection velocities estimated through semi-empirical and theoretical models proposed in the literature are compared with the particle behaviour in the bubble dome obtained from the two-fluid simulations. The results obtained here indicate that the theory based on the potential flow around a cylinder provides a more accurate prediction for the particle velocities in erupting bubbles than semi-empirical relations. For the data reported here it has been found that the velocity of particles in the bubble dome forms an angle with the vertical direction that is twice the angle formed by the radial direction. This observation is contrary to standard models of 2D bubbles, which assume that the particles are ejected radially outwards from the dome.
\end{abstract}

\section{Introduction}

Fluidized beds have various applications in industry, such as fluid catalytic cracking (FCC), gasification, combustion of solid fuels, and Fischer-Tropsch synthesis [1]. Despite the fact that fluidized beds have been used in industry since the 1920s and good progress has been made in numerical simulation of these systems using twofluid [2] or discrete element models [3], some aspects of fluidized bed dynamics are still far from fully understood.

One aspect worthy of further study is the behaviour at the top of the bed, that is, bubble eruption and collapse, and the associated ejection, elutriation or entrainment of particles. An understanding of the underlying physics of these processes is not only important from an academic point of view but is also of industrial relevance. The rate of particle elutriation affects the operation of a fluidized bed reactor, and the height of particle entrainment is an important design parameter. It has been suggested that particles are ejected into the freeboard either from the roof $[4,5]$ or the wake of a bubble [6]. Pemberton and Davidson [7] emphasised

\footnotetext{
* Corresponding author. Tel.: +34 916246211.

E-mail address: fhjimene@ing.uc3m.es (F. Hernández-Jiménez).
}

the importance of the effect of the walls on the ejection mechanism, finding that in a two-dimensional (2D) bed, ejection from the roof of the bubble dominated, whereas in a three-dimensional (3D) bed ejection from the wake of the bubble was favoured for sufficiently high gas velocities. The pattern in which bubbles erupt can also influence whether particles are ejected from the wake or the roof of a bubble, and the maximum height reached by the ejected particles [8,9]. Hatano and Ishida [9] divided the patterns of bubbles bursting at the top of a fluidized bed into four categories: (1) isolated bubbles, (2) successively rising bubbles, (3) coalescing bubbles, and (4) successively coalescing bubbles. In the case of the eruption of an isolated bubble, particles are ejected from the roof of the bubble, whereas particles are ejected from the wake of the leading bubble in the case of coalescing bubbles $[8,9]$.

Numerical modelling of fluidized bed systems has advanced significantly over the last two decades, the most popular modelling approaches being the Eulerian-Eulerian and the Eulerian-Lagrangian models. In the development and application of these techniques, careful validation with either experimental data or theoretical models is required [10]. In the last years several reports have appeared in this field comparing two-fluid simulations with experimental data, such as [11,12]. 


\begin{tabular}{|c|c|}
\hline \multicolumn{2}{|c|}{ Nomenclature } \\
\hline$A$ & bubble area $\left(\mathrm{m}^{2}\right)$ \\
\hline$D_{\text {eq }}$ & bubble equivalent diameter $(\mathrm{m})$ \\
\hline$K_{\mathrm{gs}}$ & gas-solid momentum exchange $\left(\mathrm{kg} /\left(\mathrm{s} \mathrm{m}^{3}\right)\right)$ \\
\hline$R$ & bubble equivalent radius ( $\mathrm{m}$ ) \\
\hline$U_{\mathrm{b}}$ & bubble velocity $(\mathrm{m} / \mathrm{s})$ \\
\hline$U_{\mathrm{gr}}$ & bubble growth velocity $(\mathrm{m} / \mathrm{s})$ \\
\hline$U_{\mathrm{p}, \mathrm{b}}$ & particle velocity related to bubble velocity $(\mathrm{m} / \mathrm{s})$ \\
\hline$U_{\mathrm{p}, \mathrm{g}}$ & particle velocity related to bubble growth $(\mathrm{m} / \mathrm{s})$ \\
\hline$U$ & particle horizontal velocity $(\mathrm{m} / \mathrm{s})$ \\
\hline$V$ & particle vertical velocity $(\mathrm{m} / \mathrm{s})$ \\
\hline$\vec{V}$ & particle velocity $(\mathrm{m} / \mathrm{s})$ \\
\hline$g$ & gravity vector $\left(\mathrm{m} / \mathrm{s}^{2}\right)$ \\
\hline$p$ & pressure $(\mathrm{Pa})$ \\
\hline$\vec{v}_{\mathrm{g}}$ & gas velocity $(\mathrm{m} / \mathrm{s})$ \\
\hline$\vec{v}_{\mathrm{s}}$ & solids velocity $(\mathrm{m} / \mathrm{s})$ \\
\hline$x$ & horizontal coordinate $(\mathrm{m})$ \\
\hline$y$ & vertical coordinate $(\mathrm{m})$ \\
\hline \multicolumn{2}{|c|}{ Greek letters } \\
\hline$\alpha_{\mathrm{s}}$ & solids volume fraction $(-)$ \\
\hline$\alpha_{\mathrm{g}}$ & voidage $(-)$ \\
\hline$\beta^{5}$ & velocity vector angle in the dome $\left(^{\circ}\right)$ \\
\hline$\rho_{\mathrm{S}}$ & solids density $\left(\mathrm{kg} / \mathrm{m}^{3}\right)$ \\
\hline$\rho_{\mathrm{g}}$ & gas density $\left(\mathrm{kg} / \mathrm{m}^{3}\right)$ \\
\hline$\omega$ & vorticity $(1 / \mathrm{s})$ \\
\hline$\theta$ & eruption angle $\left(^{\circ}\right)$ \\
\hline$\theta_{\mathrm{b}}$ & direction of bubble rise $\left(^{\circ}\right)$ \\
\hline$\theta_{\max }$ & maximun eruption angle $\left({ }^{\circ}\right)$ \\
\hline$\theta_{\min }$ & minimun eruption angle $\left({ }^{\circ}\right)$ \\
\hline & velocity potential flow $\left(\mathrm{m}^{2} / \mathrm{s}\right)$ \\
\hline$\Theta$ & granular temperature $(\mathrm{J} / \mathrm{kg})$ \\
\hline
\end{tabular}

In the Eulerian-Lagrangian approach the particles are treated as single entities, the motion of which is governed by Newton's second law. A Lagrangian simulation of each particle trajectory is coupled with an Eulerian simulation of the bulk gas flow. The interaction between the solid and gaseous phases is computed through semi-empirical closure models [13]. Although very promising, the Eulerian-Lagrangian approach is very computationally expensive and is, therefore, currently unable to simulate the vast number of particles encountered in medium- or large-scale fluidized beds.

In two-fluid modelling of fluidized beds $[2,14]$ the gas and solid phases are treated as two interpenetrating continua using the conservation equations of fluids. As in the case of the Eulerian-Lagrangian approach, the two-fluid simulation of fluidized beds requires the use of closure models for the gas-solid interaction. However, since the particle motion is not modelled in detail, the two-fluid model also requires closure models for the particle-particle interactions. These closure relationships may be empirical in nature or may be based on theoretical relations that are linked to the kinetic theory of gases through the concept of granular temperature [2].

Now, it is turned to experimental studies, using Particle Image Velocimetry (PIV), investigating the eruption of solids in fluidized beds. Santana et al. [15] studied the mechanism of solids ejection in a bubbling fluidized bed of $5 \mathrm{~mm}$ thickness filled with sand particles of $0.3 \mathrm{~mm}$ mean diameter and fluidized with air at superficial velocity of $U=2.8 \times U_{\mathrm{mf}}$. They found good agreement between their measurements and the empirical model proposed by Fung and Hamdullahpur [16]. Using the same bed, Almendros-Ibáñez et al. [17] continued this work for a range of fluidization velocities (i.e. from 2 to 4 times $U_{\mathrm{mf}}$ ) and modified the Fung and Ham- dullahpur model by taking into account the bubble velocity, the bubble growth, and the bubble rise direction. Later, Müller et al. [18] extended the work of Santana et al. [15] by studying the vorticity of the particulate phase surrounding a bubble and applying the potential flow theory [7] to predict the particle velocities in the dome of the bubble. For that study, Müller et al. [18] employed a bed of $10 \mathrm{~mm}$ thickness filled with either sand or aluminium oxide particles with mean diameters of approximately $0.6 \mathrm{~mm}$ and $0.5 \mathrm{~mm}$, respectively. Superficial velocities covering a range from 1.2 to 1.96 times $U_{\mathrm{mf}}$ were studied. Although the potential flow theory was originally developed for isolated bubbles ascending in a fluidized bed, the results reported in Ref. [7] showed that potential flow theory can also be used to predict the particle velocity in the dome of an erupting bubble.

Although there are differences between laboratory controlled $2 \mathrm{D}$ beds and real 3D beds, general theories describing the bubble behaviour before eruption in 2D situations have proved to be extensible to 3D domains by merely adding a third dimension in the resulting models. This is, for example, the case of potential flow theory [19]. Furthermore, the mechanism of particle ejection in 3D beds in which the superficial gas velocity is less than 10 or 15 times $U_{\mathrm{mf}}$ is very similar to the mechanism that dominants in $2 \mathrm{D}$ beds, i.e. particles are ejected predominantly from the roof of bursting bubbles [7]. Therefore, it is believed that studies performed in 2D beds are a valuable tool for assessing and developing bubble eruption models. However, it should be stressed that other phenomena in 2D fluidized bed studies may not easily transfered to 3D beds.

The present work reports two-fluid simulations of a 2D bed filled with particles group B of Geldart's classification and fluidized with air. The main objective of this study is to test the ability of such simulations to reproduce realistically the particle velocity during bubble eruption, as well as to compare the results with (i) experimental data and (ii) existing semi-empirical and theoretical models for particle ejection. In the first part of the work, the particle velocities around an isolated bubble in the centre of the simulated bed are compared with results derived from the potential flow theory, which is commonly accepted as a good representation of the particle velocity around bubbles far from walls or from other bubbles [1]. The effect of a second, smaller bubble on the simulated velocity profile around a bubble is also studied. In the second part of the work, the velocity profiles of particles in the dome of an erupting bubble are analysed and compared with experimental PIV data together with the velocity profiles predicted by the particle ejection models of Fung and Hamdullahpur [16], Almedros-Ibáñez et al. [17], and Müller et al. [18]. Such a comprehensive comparison has not been reported in the literature before and allows a critical evaluation of various particle ejection models. Additionally, once the capabilities of the two-fluid model have been verified in the framework of isolated and erupting bubbles, the relationship between the instantaneous gas and particle velocities in the bubble dome is investigated using results from the two-fluid simulation. Such coupled information regarding the two phases (gas and solids) is difficult to obtain experimentally, which confers an added value to the simulation results.

\section{Simulated system}

In this work the bubble dynamics were studied in two fluidized beds. Table 1 summarises the most important parameters for these two systems. Case 2 was chosen to match the experimental system reported by Müller et al. [18]. For both systems the gas inlet velocity was set to be $U=1.25 \times U_{\mathrm{mf}}$ such that the bed operates in a bubbling regime with only a small number of bubbles present. This ensures the existence of isolated bubbles within the centre of the bed, which are required for this study. Fig. 1 shows contours of solids volume 
Table 1

Simulation parameters.

\begin{tabular}{lcc}
\hline Parameter & Case 1 & Case 2 \\
\hline Particle density $\left(\mathrm{kg} / \mathrm{m}^{3}\right)$ & 1000 & 2600 \\
Gas density $\left(\mathrm{kg} / \mathrm{m}^{3}\right)$ & 1.2 & 1.2 \\
Gas viscosity $(\mathrm{Pa} \mathrm{s})$ & $1.8 \mathrm{e}-5$ & $1.8 \mathrm{e}-5$ \\
Particle diameter $(\mathrm{mm})$ & 1.2 & 0.6 \\
Minimun fluidization velocity $(\mathrm{m} / \mathrm{s})$ & 0.3 & 0.225 \\
Bed width $(\mathrm{m})$ & 0.2 & 0.2 \\
Bed height $(\mathrm{m})$ & 0.5 & 0.5 \\
Static bed height $(\mathrm{m})$ & 0.27 & 0.27 \\
Initial voidage $(-)$ & 0.4 & 0.4 \\
Superficial gas velocity $(\mathrm{m} / \mathrm{s})$ & 0.375 & 0.283 \\
Restitution coeffcient $(-)$ & 0.9 & 0.9 \\
Angle of internal friction $\left({ }^{\circ}\right)$ & 30 & 30 \\
\hline
\end{tabular}

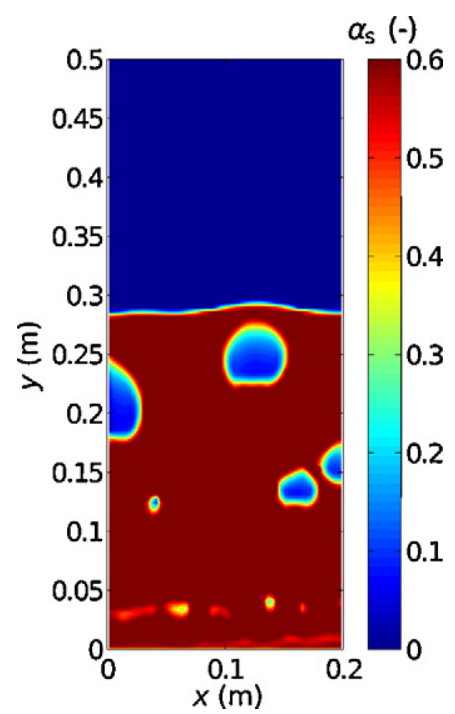

Fig. 1. Snapshot of the particle volume fraction in the 2D fluidized bed simulated (case 1 ).

fraction for a snapshot of simulation case 1. A Cartesian coordinate system is employed in this work, with the $x$ and $y$ axes aligned along the horizontal and vertical directions respectively.

\section{Two-fluid model}

\subsection{General equations}

The two-fluid model, based on the conservation equations of mass, momentum and granular temperature, was solved using the MFIX code (Multiphase Flow with Interphase eXchanges) $[20,21]$. The kinetic theory of granular flow, which characterized the stochastic fluctuations of the solids kinetic energy, was used for the closure of the solids stress terms. The governing equations are summarised summarised in the following lines. Mass conservation of the gas ( $\mathrm{g}$ ) and solid ( $\mathrm{s}$ ) phases:

$\frac{\partial}{\partial t}\left(\alpha_{\mathrm{g}} \rho_{\mathrm{g}}\right)+\nabla \cdot\left(\alpha_{\mathrm{g}} \rho_{\mathrm{g}} \vec{v}_{\mathrm{g}}\right)=0$

$\frac{\partial}{\partial t}\left(\alpha_{\mathrm{s}} \rho_{\mathrm{s}}\right)+\nabla \cdot\left(\alpha_{\mathrm{s}} \rho_{\mathrm{s}} \vec{v}_{\mathrm{s}}\right)=0$

Momentum conservation of the gas and solids phases:

$\frac{\partial}{\partial t}\left(\alpha_{\mathrm{g}} \rho_{\mathrm{g}} \vec{v}_{\mathrm{g}}\right)+\nabla\left(\alpha_{\mathrm{g}} \rho_{\mathrm{g}} \vec{v}_{\mathrm{g}}^{2}\right)=-\alpha_{\mathrm{g}} \nabla p+\nabla \overline{\overline{\tau_{\mathrm{g}}}}+\alpha_{\mathrm{g}} \rho_{\mathrm{g}} \overrightarrow{\mathrm{g}}-K_{\mathrm{gs}}\left(\vec{v}_{\mathrm{g}}-\vec{v}_{\mathrm{s}}\right)$

$\frac{\partial}{\partial t}\left(\alpha_{\mathrm{s}} \rho_{\mathrm{s}} \vec{v}_{\mathrm{s}}\right)+\nabla\left(\alpha_{\mathrm{s}} \rho_{\mathrm{s}} \vec{v}_{\mathrm{s}}^{2}\right)=-\alpha_{\mathrm{s}} \nabla p-\nabla p_{\mathrm{s}}+\nabla \overline{\overline{\tau_{\mathrm{s}}}}+\alpha_{\mathrm{s}} \rho_{\mathrm{s}} \vec{g}+K_{\mathrm{gs}}\left(\vec{v}_{\mathrm{g}}-\vec{v}_{\mathrm{s}}\right)$
Granular temperature, $\Theta$, conservation equation:

$$
\begin{aligned}
& \frac{3}{2}\left(\frac{\partial}{\partial t}\left(\rho_{\mathrm{s}} \alpha_{\mathrm{s}} \Theta\right)+\nabla\left(\rho_{\mathrm{s}} \alpha_{\mathrm{s}} \vec{v}_{\mathrm{s}} \Theta\right)\right) \\
& =\left(-p_{\mathrm{s}} \overline{\bar{I}}+\overline{\overline{\tau_{\mathrm{s}}}}\right): \nabla \vec{v}_{\mathrm{s}}+\nabla\left(k_{\Theta} \nabla \Theta\right)-\gamma_{\Theta}-3 K_{\mathrm{gs}} \Theta
\end{aligned}
$$

where $p_{\mathrm{s}}$ is the solids pressure, $\overline{\overline{\tau_{i}}}=\alpha_{i} \mu_{i}\left(\nabla \vec{v}_{i}+\nabla \vec{v}_{i}^{T}\right)+\alpha_{i}\left(\lambda_{i}-\right.$ $\left.(2 / 3) \mu_{i}\right) \nabla \cdot \vec{v}_{i} \overline{\bar{I}}$ is the strain tensor for phase $i,\left(-p_{s} \overline{\bar{I}}+\overline{\overline{\tau_{s}}}: \nabla \vec{v}_{\mathrm{s}}\right)$ is the generation of $\Theta$ by the solids stresses, $k_{\Theta} \nabla \Theta$ is the diffusion of $\Theta$ energy, $\gamma_{\Theta}$ is the collisional dissipation of $\Theta$ and $3 K_{\mathrm{gs}} \Theta$ is the transfer of random kinetic energy between the solids and the gas. The closure expressions for the model can be found in Ref. [21].

A second order accurate scheme was used to discretise the convective derivatives. For simulation case 1 , the $2 \mathrm{D}$ computational domain was discretised using square cells of $3.6 \mathrm{~mm}$ length in a mesh of 7645 nodes. Thus, the cell size was 3 times the particle diameter. For simulation case 2 , square cells of $2.5 \mathrm{~mm}$ length were chosen due to the smaller particle size used in this case, resulting in a mesh of 15288 nodes. The initial time step was set to $0.5 \times 10^{-5} \mathrm{~s}$ to ensure convergence of the equation system during the first seconds of the simulation. A uniform and steady velocity profile was chosen at the inlet boundary and a fixed pressure boundary condition was chosen at the top of the freeboard. The lateral walls were modelled as no-slip walls for both phases. It is felt that the lateral boundary condition does not have a strong influence on the motion of an isolated bubble in the centre of the bed. The initial solids volume fraction was set to 0.6 .

The solids volume fraction and solids velocity were recorded every $0.005 \mathrm{~s}$ to provide the temporal resolution required to study bubble eruption events. In order to study bubble motion, it is necessary to distinguish between bubbles and the emulsion phase. This is done by setting a cutoff value for the solids volume fraction equal to $\alpha_{\mathrm{s}}=0.3$, which is the arithmetic mean of the maximum and minimum solids volume fractions in the simulated bed. Any region in which the solids volume fraction is less than 0.3 is defined to be a bubble.

\subsection{Force correlation used}

The drag force correlation used in this study is the extension of the Hill and Koch drag law [22,23], proposed by Benyahia et al. [24]. In this correlation the friction coefficient is defined as:

$K_{\mathrm{gs}}=18 \mu_{\mathrm{g}}\left(1-\alpha_{\mathrm{s}}\right)^{2} \alpha_{\mathrm{s}} \frac{F}{d_{\mathrm{p}}^{2}}$

where $\mu_{\mathrm{g}}$ is the gas viscosity, $\alpha_{\mathrm{s}}$ is the solids volume fraction, $d_{\mathrm{p}}$ is the particle diameter, and $F$ is the dimensionless drag force, which is expressed as a function of the particle Reynolds number $R e=$ $\left(\rho_{\mathrm{g}}\left(1-\alpha_{\mathrm{s}}\right)\left|v_{\mathrm{g}}-v_{\mathrm{s}}\right| d_{\mathrm{p}} / 2 \mu_{\mathrm{g}}\right)$, and a set of parameters as follows:

$F=\frac{1+3}{8 \operatorname{Re}} \quad \alpha_{\mathrm{s}} \leq 0.01, \quad \operatorname{Re} \leq \frac{\left(F_{2}-1\right)}{\left(3 / 8-F_{3}\right)}$

$F=F_{0}+F_{1} \operatorname{Re}^{2} \quad \alpha_{\mathrm{s}}>0.01, \quad \operatorname{Re} \leq \frac{F_{3}+\sqrt{F_{3}^{2}-4 F_{1}\left(F_{0}-F_{2}\right)}}{2 F_{1}}$

$F=F_{2}+F_{3} \operatorname{Re}\left\{\begin{array}{ll}\alpha_{\mathrm{s}} \leq 0.01, & \operatorname{Re}>\frac{F_{2}-1}{3 / 8-8 F_{3}} \\ \alpha_{\mathrm{s}}>0.01, & \operatorname{Re}>\frac{F_{3}+\sqrt{F_{3}^{2}-4 F_{1}\left(F_{0}-F_{2}\right)}}{2 F_{1}}\end{array}\right\}$

$F_{0}=\left\{\begin{array}{ll}(1-w) a+w b & 0.01<\alpha_{\mathrm{s}}<0.4 \\ b & \alpha_{\mathrm{s}} \geq 0.4\end{array}\right\}$ 
$F_{1}=\left\{\begin{array}{ll}\sqrt{\frac{2}{\alpha_{\mathrm{s}}} / 40} & 0.01<\alpha_{\mathrm{s}} \leq 0.1 \\ 0.11+0.00051 \exp \left(11.6 \alpha_{\mathrm{s}}\right) & \alpha_{\mathrm{s}}>0.1\end{array}\right\}$

$F_{2}=\left\{\begin{array}{ll}(1-w) c+w b & \alpha_{\mathrm{s}}<0.4 \\ b & \alpha_{\mathrm{s}} \geq 0.4\end{array}\right\}$

$F_{3}=\left\{\begin{array}{ll}0.9351 \alpha_{\mathrm{s}}+0.03667 & \alpha_{\mathrm{s}}<0.0953 \\ 0.0673+0.212 \alpha_{\mathrm{s}}+0.0232 /\left(1-\alpha_{\mathrm{s}}\right)^{5} & \alpha_{\mathrm{s}} \geq 0.0953\end{array}\right\}$

$a=\left[\frac{1+3 \sqrt{\alpha_{\mathrm{s}} / 2}+(135 / 64) \alpha_{\mathrm{s}} \ln \left(\alpha_{\mathrm{s}}\right)+17.14 \alpha_{\mathrm{s}}}{1+0.681 \alpha_{\mathrm{s}}-8.48 \alpha_{\mathrm{s}}^{2}+8.16 \alpha_{\mathrm{s}}^{3}}\right]$

$b=\left[10 \frac{\alpha_{\mathrm{s}}}{\left(1-\alpha_{\mathrm{s}}\right)^{3}}\right]$

$c=\left[\frac{1+3 \sqrt{\alpha_{\mathrm{s}} / 2}+(135 / 64) \alpha_{\mathrm{s}} \ln \left(\alpha_{\mathrm{s}}\right)+17.89 \alpha_{\mathrm{s}}}{1+0.681 \alpha_{\mathrm{s}}-11.03 \alpha_{\mathrm{s}}^{2}+15.41 \alpha_{\mathrm{s}}^{3}}\right]$

$w=e^{\left(-10\left(0.4-\alpha_{\mathrm{s}}\right) / \alpha_{\mathrm{s}}\right)}$

\section{Theoretical models for particle ejection}

The bubble eruption results obtained from the simulations are compared to three bubble eruption models: (i) potential flow theory [18], (ii) the Fung and Hamdullahpur model [16], and (iii) the Almendros-Ibáñez model [17].

Pemberton and Davidson [7] used the potential flow theory to predict the particle flow in the dome formed by an erupting bubble. Müller et al. [18] extended this theory to predict the velocity of particles at the top of the dome formed by an erupting bubble. The velocity potential, $\Phi$, is governed by Laplace's equation:

$\nabla^{2} \Phi=0$

For a 2D system, the velocities in the $x$ and $y$ directions are given by:

$U=\frac{\partial \Phi}{\partial x}$

$V=\frac{\partial \Phi}{\partial y}$

As a first approximation, the bubble in a $2 \mathrm{D}$ bed is assumed to be of cylindrical shape with a radius $R$ and velocity $U_{\mathrm{b}}$. The velocity vector of the bubble centroid forms an angle $\theta_{\mathrm{b}}$ with the positive vertical direction [25]. In this work the angle $\theta_{\mathrm{b}}$ shall be termed the direction of bubble rise. The velocity potential around a cylinder is given by [19] as:

$\Phi_{x y}=-U_{\mathrm{b}}\left(\frac{R^{2}}{x^{2}+y^{2}}\right)\left(y \cos \left(\theta_{\mathrm{b}}\right)+x \sin \left(\theta_{\mathrm{b}}\right)\right)$

Here the coordinate pair $(x, y)$ describe the distance from the centre of the cylinder in the horizontal and vertical directions. Using Eqs. (8) and (9) the velocities in the $x$ and $y$ directions are given by:

$U=\left(\frac{U_{\mathrm{b}} R^{2}}{x^{2}+y^{2}}\right)\left(\frac{2 x\left(y \cos \left(\theta_{\mathrm{b}}\right)+x \sin \left(\theta_{\mathrm{b}}\right)\right)}{x^{2}+y^{2}}-\sin \left(\theta_{\mathrm{b}}\right)\right)$

$V=\left(\frac{U_{\mathrm{b}} R^{2}}{x^{2}+y^{2}}\right)\left(\frac{2 y\left(y \cos \left(\theta_{\mathrm{b}}\right)+x \sin \left(\theta_{\mathrm{b}}\right)\right)}{x^{2}+y^{2}}-\cos \left(\theta_{\mathrm{b}}\right)\right)$
If the bubble ascends in vertical direction, $\theta_{\mathrm{b}}=0$, and the expressions (10), (11) and (12) can be simplified to give:

$\Phi_{x y}=-U_{\mathrm{b}} y\left(\frac{R^{2}}{x^{2}+y^{2}}\right)$

$U=2 U_{\mathrm{b}} x y\left(\frac{R}{x^{2}+y^{2}}\right)^{2}$

$V=U_{\mathrm{b}}\left(y^{2}-x^{2}\right)\left(\frac{R}{x^{2}+y^{2}}\right)^{2}$

Turning now to bubble eruption models, Fung and Hamdullahpur [16] assumed a linear decrease of the particle velocity with the eruption angle, $\theta$ :

$\frac{\vec{V}}{V_{\max }}=\left(1-\frac{|\theta|}{\theta_{\max }}\right) \vec{e}_{\mathrm{r}}$

where $V_{\max }$ is the maximum vertical velocity of the particles in the dome of the bubble, $\vec{V}$ is the particle velocity vector, $\theta_{\max }$ is the maximum value of the eruption angle and $\vec{e}_{\mathrm{r}}$ is the unit vector in the radial direction relative to the centroid of the bubble. The eruption angle, $\theta$, is the angle that defines the position of any point in the bubble dome, and can cover a range from $-90^{\circ}$ to $90^{\circ}$. The eruption angle is defined to be $0^{\circ}$ at the top of the bubble, as indicated in Fig. 2.

Almendros-Ibáñez et al. [17] modified the model of Fung and Hamdullahpur [16] by including the bubble velocity, the bubble growth, and the direction of bubble rise. They described the particle velocity using the expression

$\vec{V}(\theta)=\vec{U}_{\mathrm{p}, \mathrm{b}}(\theta)+\vec{U}_{\mathrm{p}, \mathrm{gr}}(\theta)$

where $\vec{V}$ is the particle velocity and the added subscripts $b$ and $\mathrm{gr}$ indicate the contributions from the velocity of the centroid of the bubble and the bubble growth, respectively. These terms were expressed as:

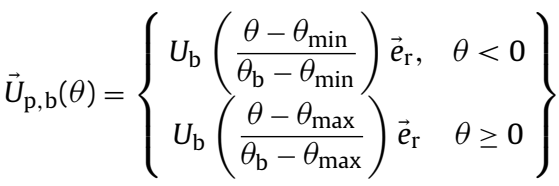

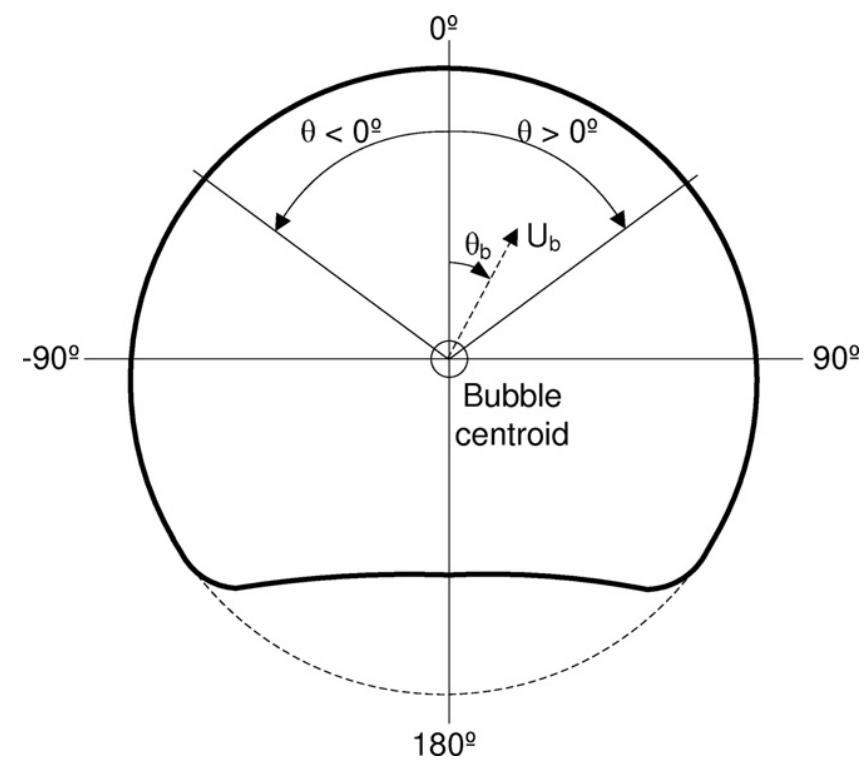

Fig. 2. Sketch of a bubble showing the definition of the eruption angle, $\theta$, and the direction of bubble rise, $\theta_{\mathrm{b}}$ 
$\vec{U}_{\mathrm{p}, \mathrm{gr}}(\theta)=\left\{\begin{array}{cc}U_{\mathrm{gr}}\left(2-\sin \left(\theta_{\mathrm{b}}\right)\left|\sin \left(\theta_{\mathrm{b}}\right)\right|\right)\left(1-\frac{\theta-\theta_{\min }}{\theta_{\mathrm{b}}-\theta_{\min }}\right) \vec{e}_{\mathrm{r}}, & \theta<0 \\ U_{\mathrm{gr}}\left(2+\sin \left(\theta_{\mathrm{b}}\right)\left|\sin \left(\theta_{\mathrm{b}}\right)\right|\right)\left(1-\frac{\theta-\theta_{\max }}{\theta_{\mathrm{b}}-\theta_{\max }}\right) \vec{e}_{\mathrm{r}}, & \theta \geq 0\end{array}\right\}$

where $\theta$ is the eruption angle and $\theta_{\mathrm{b}}$ is the angle between the bubble rise velocity vector and the vertical direction. As Eqs. (18) and (19) are applicable for non-symmetrical domes, the positive and negative limits of the eruption angle, $\theta_{\max }$ and $\theta_{\min }$ respectively, can have different values. The bubble velocity in this model is calculated at the instant when the bubble breaks through the dome. This instant is difficult to define in the two-fluid model due to the continuous character of the simulated solids-phase. In the present work the process of bubble eruption starts when the vertical velocities of the particles within the bubble dome begin to decrease. For the cases considered here, $\theta_{\mathrm{b}}$ is very close to $0^{\circ}$, i.e. the ascent of the bubbles is almost vertical. In Eq. (21), the bubble growth velocity can be defined as follows:

$U_{\mathrm{gr}}=\frac{1}{2} \frac{\partial D_{\mathrm{eq}}}{\partial t}$

The model described by Eqs. (17)-(20) states that the maximum particle velocity at the dome of an erupting bubble is equal to the bubble velocity. However, this leads to an overestimation of the maximum particle velocities when compared to the results of the two-fluid simulation because the particle velocities in the bubble dome are smaller than the velocity of the centroid of the bubble. Thus, a modification of the particle ejection model of AlmendrosIbáñez et al. [17] is suggested. Instead of using the velocity of the bubble centroid in Eq. (18), the velocity $U_{\mathrm{b}}$ shall be obtained from the simulation results for the bubble dome at the position $\theta=0^{\circ}$, as is the case for $V_{\max }$ in Eq. (16). This leads to:

$\vec{U}_{\mathrm{p}, \mathrm{b}}(\theta)=\left\{\begin{array}{cc}V_{\max }\left(\frac{\theta-\theta_{\min }}{\theta_{\mathrm{b}}-\theta_{\min }}\right), & \theta<0 \\ V_{\max }\left(\frac{\theta-\theta_{\max }}{\theta_{\mathrm{b}}-\theta_{\max }}\right), & \theta \geq 0\end{array}\right\}$

It is important to note that the models of Fung and Hamdullahpur [16] and Almendros-Ibáñez et al. [17] assume that particles are ejected in the radial direction. However, this is not the case in the potential flow model described by Eq. (13).

In order to compare the results of the two-fluid simulations with the models described above, the velocity of bubbles within the simulations must be obtained. In this work the bubble velocity is estimated based on the displacement of the centre of mass of the bubble calculated over ten time steps. The diameter of the bubble is assumed to be the diameter of a circle with an area equal to that of the bubble, $D_{\text {eq }}=\sqrt{4 A / \pi}$.

\section{Results and discussion}

\subsection{Isolated bubbles}

The first part of the results focuses on comparing particle velocities extracted from the two-fluid model for an isolated bubble in the middle of the bed with the velocity predicted by the potential flow theory, Eq. (13). Here, an arbitrarily selected bubble is analysed for each of the two cases summarised in Table 1. During the course of this work several bubbles have been analysed for each simulation case. Similar behaviour was observed for all of the bubbles studied. Therefore, it is felt that the bubbles presented here are representative of the bubbles that have been analysed.

The bubble studied for simulation case 1 has an equivalent diameter of $D_{\text {eq }}=3.3 \times 10^{-2} \mathrm{~m}$ and a rise velocity of $U_{\mathrm{b}}=0.369 \mathrm{~m} / \mathrm{s}$. The direction of bubble rise is approximately $\theta_{\mathrm{b}}=0^{\circ}$. The bubble is as isolated as possible, with no other bubbles in its surroundings and as far as possible from the freeboard and the side walls. Fig. 3 shows three snapshots of the isolated bubble consisting of contour maps of the solids volume fraction, the solids velocity, and the vorticity, following the analysis performed in Ref. [18]. Arrows representing the solids velocity vectors have been superimposed on the velocity and vorticity maps. For clarity only every second vector has been plotted. For 2D systems, the vorticity of the particulate phase is given by:

$\omega=\left(\frac{\partial V}{\partial x}-\frac{\partial U}{\partial y}\right)$

where the partial derivatives are calculated from the simulation data using a second order central difference scheme involving a $3 \times 3$ matrix of mesh nodes. Information about the vorticity of a fluidized bed is highly relevant because most models and correlations are based on potential flow theory and can, therefore, be compromised if $\omega$ is not close to zero in the emulsion phase.

The maps of solids fraction and velocity in Fig. 3 suggest qualitative agreement with the potential flow theory: The bubble is nearly circular, the highest solids velocities are located at the sides of the bubble, and the bubble wake is restricted to a small region in the lower part of the bubble. Both the velocity vectors and vorticity in the wake of the bubble indicate that the largest vorticity is confined to the bubble wake and that the vorticity rapidly decreases outside of this region. Fig. 4 plots the simulation results for the vertical and horizontal velocities of the solid phase at points around a circle surrounding the contour defining the bubble (i.e. the contour defined by $\alpha_{\mathrm{s}}=0.3$ in Fig. 3). For comparison purposed, the solid phase velocities predicted by potential flow theory around a cylinder of radius $R=D_{\mathrm{eq}} / 2$ are also shown in Fig. 4 . The horizontal axis in Fig. 4 is the angle $\theta$ of each point around the circle. As in the case of the particle ejection models described in the previous section, $\theta$ starts at the bubble top and increases in the clockwise direction. The results shown in Fig. 4 for both the horizontal and vertical velocities show good quantitative agreement around the whole bubble perimeter, particularly at the bubble top defined by $-90^{\circ}<\theta<90^{\circ}$. Even in the bubble wake, where the deviation of the bubble shape from a circle is expected to be greatest, the agreement of both the shape and magnitude of the velocity profiles is remarkable.

The bubble analysed for case 2 has a similar size and velocity to the bubble considered above. Specifically, the bubble has an equivalent diameter of $D_{\mathrm{eq}}=3.5 \times 10^{-2} \mathrm{~m}$ and a rise velocity of $U_{\mathrm{b}}=0.327 \mathrm{~m} / \mathrm{s}$. The direction of bubble rise is approximately $\theta_{\mathrm{b}}=0^{\circ}$. Fig. 5 shows contour maps of the solids volume fraction, the velocity of the solids and the vorticity of the solid phase for this bubble. These data indicate that this bubble is not isolated and that there is a second, smaller bubble to the right of it. This smaller bubble interacts with the larger bubble and disturbs the flow pattern around it.

Fig. 6 shows the horizontal and vertical velocities of the solid phase at points on a circle surrounding the large bubble show in Fig. 5. For comparison, the velocities predicted by potential flow theory are also plotted. The simulated velocities circumscribing the selected bubble show good agreement with the predictions from potential flow theory. It is interesting to note that the influence of the small bubble on the velocities of the solid phase encircling the large bubble is restricted to a small region close to the location of the small bubble. This suggests that the potential flow theory may be a robust model for predicting the flow pattern around bubbles and may be able to provide useful information in non-ideal cases, such as case 2 .

Comparing the velocity profiles shown in Figs. 4 and 6, it is evident that both cases share a similar, small deviation from the potential flow model. In the upper half of the bubble, the region of 


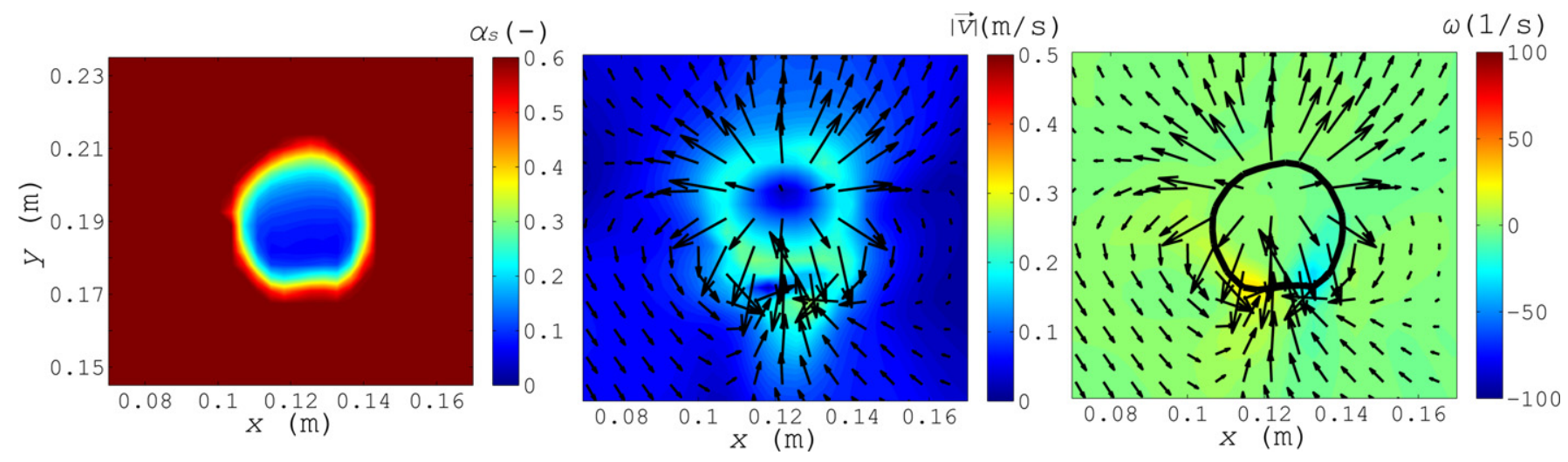

Fig. 3. Bubble contours for case 1 : solids volume fraction (-), solids velocity (m/s) and vorticity $(1 / \mathrm{s}) . U_{\mathrm{b}}=0.369 \mathrm{~m} / \mathrm{s}$.
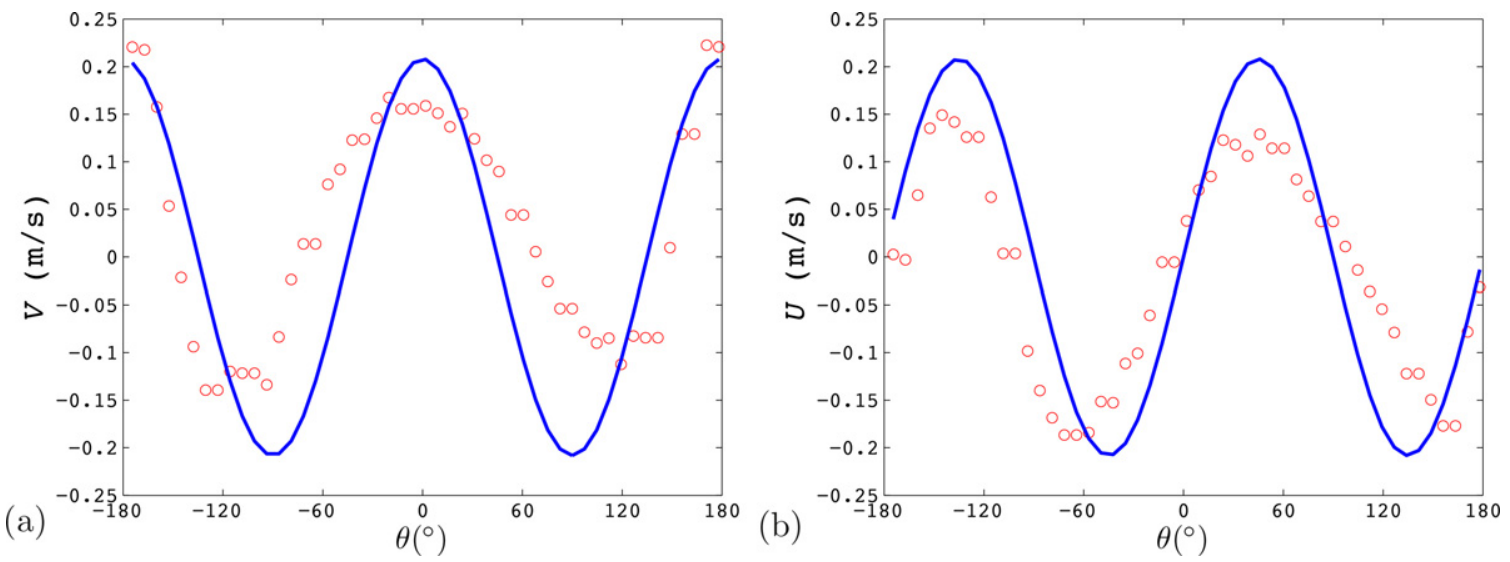

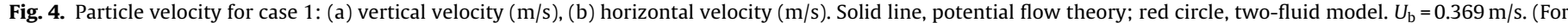
interpretation of the references to color in this figure legend, the reader is referred to the web version of the article.)

positive vertical velocity obtained from the simulation is broader than that predicted by potential flow theory (i.e. from $\theta=-45^{\circ}$ to $45^{\circ}$ ). In the lower half of the bubble, however, the region of positive vertical velocity is smaller than predicted by the potential flow profiles. This phenomena may be explained by recognising that the bubble is not a perfect circle and, thus, the centre of mass of the bubble used for the calculation of $\theta$ in the velocity profiles is placed slightly above the real centre of the circle that encloses both the bubble and its wake. However, identifying the circle that encloses the bubble and its wake is a somewhat subjective operation. For this reason, the bubbles in this work shall be represented by a circle centred at the centre of mass of the bubble.

\subsection{Bubble eruption}

In this section results obtained from the two-fluid model simulations for the vertical and horizontal velocities of particles in the dome (i.e. the roof) of erupting bubbles are compared with predictions from the particle ejection models described previously. Following the methodology used above in the study of isolated bubbles, one bubble is selected at random for each of the simulation

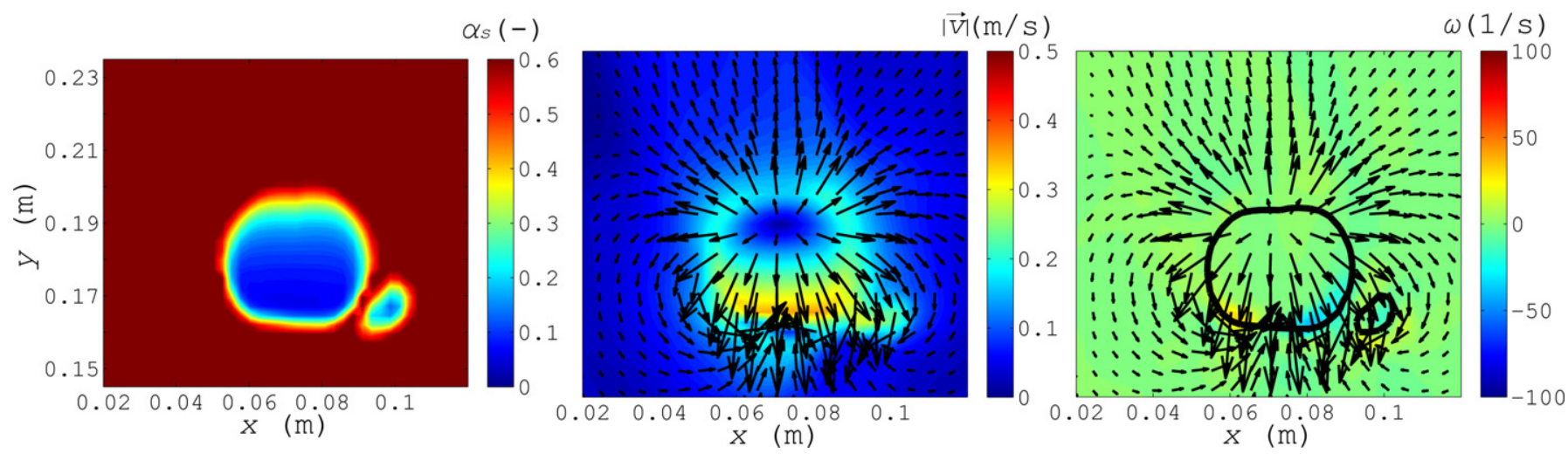

Fig. 5. Bubble contours for case 2 : solids volume fraction (-), solids velocity $(\mathrm{m} / \mathrm{s})$ and vorticity $(1 / \mathrm{s}) . U_{\mathrm{b}}=0.327 \mathrm{~m} / \mathrm{s}$. 

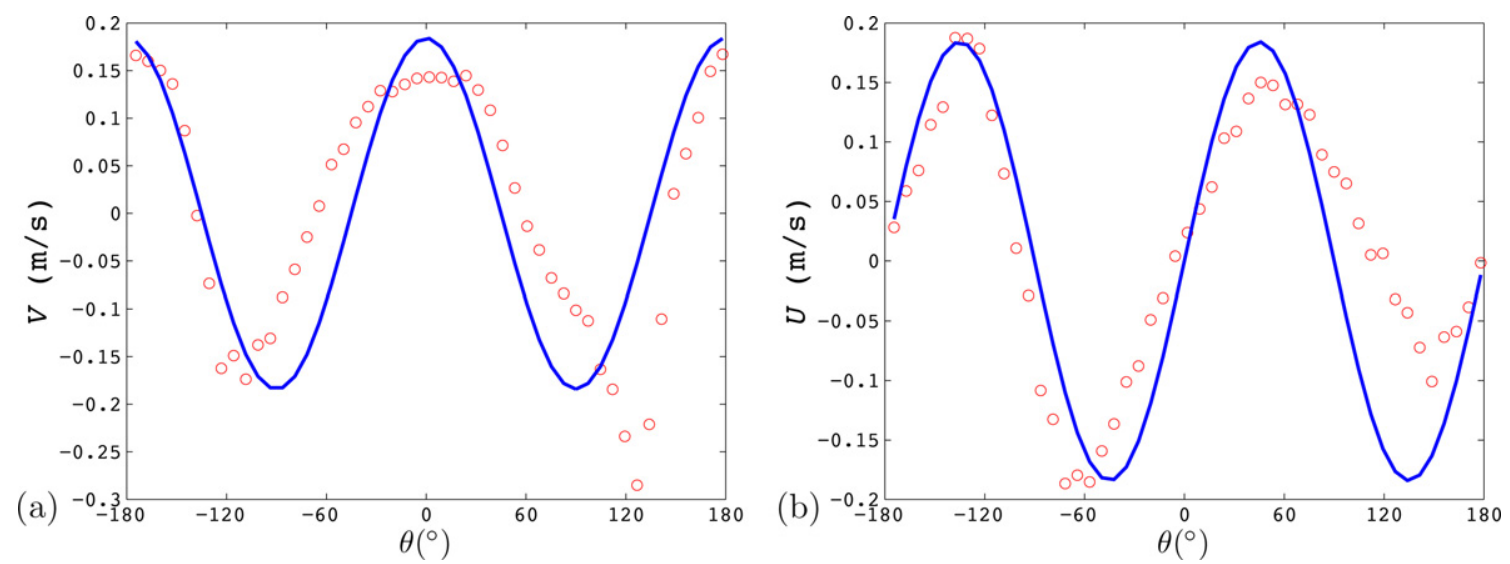

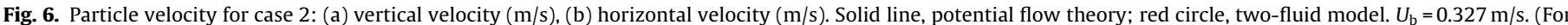
interpretation of the references to color in this figure legend, the reader is referred to the web version of the article.)

cases summarised in Table 1. Again, the bubbles analysed here are felt to be representative of the bubbles that have been analysed for each of the two simulation cases.

To allow the evolution of the particle motion during bubble eruption to be analysed, particle velocities have been obtained at various stages of the bubble eruption process. In this work bubble eruption is defined as beginning when the vertical velocities of the particles within the bubble dome begin to decrease. In the results presented here, time, $t$, is defined to be 0 at this point. Based on this definition, it is expected that, for $t \geq 0$, the particles within the bubble dome will have been ejected from the bulk of the bed and that the interactions between these particles will be greatly reduced. Consequently, these particles are assumed to follow approximately parabolic trajectories for $t \geq 0$.

Fig. 7 shows contour maps of the solids volume fraction, solids velocity and solids vorticity for an erupting bubble from simulation case 1 . Arrows indicating the solids velocity vectors have been superimposed on the velocity and vorticity plots. The first time depicted in Fig. 7 is $t=-25 \mathrm{~ms}$, that is, $25 \mathrm{~ms}$ before the bubble starts to erupt. At this time the top of the bubble is approximately $D_{\text {eq }} / 2$ below the free surface of the bed. At $t=-25 \mathrm{~ms}$ the bubble has an an equivalent diameter of $D_{\text {eq }}=4.7 \times 10^{-2} \mathrm{~m}$, a rise velocity of $U_{\mathrm{b}}=0.424 \mathrm{~m} / \mathrm{s}$, and a velocity due to bubble growth of $U_{\mathrm{g}}=5.9 \times 10^{-2} \mathrm{~m} / \mathrm{s}$. The direction of bubble rise is approximately $\theta_{\mathrm{b}}=0^{\circ}$. The velocity vectors shown in Fig. 7 indicate that between $t=25 \mathrm{~ms}$ and $t=50 \mathrm{~ms}$, the particles within the bubble dome begin to fall back towards the bed (i.e. $V<0$ ), showing that the bubble dome is starting to collapse. For $t=75 \mathrm{~ms}$, the solids volume fraction within the dome of the bubble is very low, which can be interpreted as rupture of the bubble dome.

The vorticity maps presented in Fig. 7 show that, at $t=-25 \mathrm{~ms}$, the vorticity around the bubble is close to zero, except in its wake. This is consistent with the observations made for the isolated bubbles analysed above. Interestingly, once the bubble begins to erupt, this region of high vorticity appears to remain in an approximately constant position throughout the collapse of the bubble dome.

Figs. 8 and 9 compare the velocities of particles within the dome of the bubble shown in Fig. 7, with velocities predicted by a range of bubble eruption models. The velocity profiles are plotted against the eruption angle, $\theta$, and are shown for times of $t=-25,0$ and $25 \mathrm{~ms}$. The results show that the best agreement between the ejection models and the simulation results occurs at $t=0$. At this value of $t$, all of the bubble eruption models are in good agreement with the simulation data obtained for the vertical velocities near the centre of the bubble dome (i.e. $-20^{\circ} \leq \theta \leq 20^{\circ}$ ). Considering both the vertical and horizontal velocities, the potential flow model appears to show the best agreement with the simulation data at $t=0$. The
Fung and Hamdullahpur model [16] is plotted in Figs. 8 and 9 for two different values for the maximum angle of eruption $\theta_{\max }: 50^{\circ}$ and $90^{\circ}$. These two values demonstrate the effect of varying $\theta_{\max }$ in the Fung and Hamdullahpur model [16]. It is clear from Fig. 8 that, near to the top of the bubble, $\theta_{\max }=90^{\circ}$ predicts the simulation results more accurately. However, for $\theta>20^{\circ}, \theta_{\max }=50^{\circ}$ gives a more accurate prediction of the particle velocities. Nevertheless, the model from Fung and Hamdullahpur [16] cannot predict the rounded shape of the velocity profile for either of the values of $\theta_{\max }$ studied. In Figs. 8 and 9 the model from Almendros-Ibáñez et al. [17] is presented in the modified form given by Eq. (23). The values of $\theta_{\max }$ and $\theta_{\min }$ required by this model were obtained from the bubble contours shown in Fig. 7. Close to the top of the bubble there is good agreement between this model and the simulated vertical velocities of the particles. However, this model over-predicts the vertical velocities of the particles for larger magnitudes of the eruption angle (i.e. $\theta>20^{\circ}$ ). For example, at $\theta=40^{\circ}$ Fig. 8 shows that the two-fluid simulation yields a vertical velocity of particles around 50\% smaller than the velocity derived from the modified model of Almendros-Ibáñez et al. [17]. This finding is in agreement with the relative error shown by Almendros-Ibáñez et al. [17] for their model for $\theta>50^{\circ}$ in bubbles whose mass centres are below the bed surface (i.e. case (a) and (b) in Ref. [17]). However, the relative errors reported in Almendros-Ibáñez et al. [17] were smaller in magnitude that those in Fig. 8, which may be attributed to the greater fluidization velocity and more vigorous bubbling regime studied in Ref. [17]. In this regard, due to bubble coalescence and deformation, higher particle velocities during bubble eruption can be expected if the superficial velocity of air injected into the bed is increased [16]. It should be noted that, like the Fung and Hamdullahpur model [16], the velocity profiles predicted by the model of Almendros-Ibáñez et al. [17], can be modified by varying the values of $\theta_{\max }$ and $\theta_{\min }$.

For the horizontal component of the particle velocity, $U$, Fig. 9 demonstrates that, of the models considered here, only potential flow theory is able to provide results that are comparable with the two-fluid simulation. The model of Fung and Hamdullahpur [16] and the modified Almendros-Ibáñez et al. [17] model predict qualitatively the variation of $U$ with $\theta$, but the magnitude of the velocities predicted by these models is significantly smaller than the simulation results. At large magnitudes of the eruption angle, the model of Almendros-Ibáñez et al. [17] predicts larger values for the horizontal velocity than the model of Fung and Hamdullahpur [16] because the bubble growth effect in Eq. (21) are most pronounced in the se regions. However, the inclusion of these effects does not appear to be sufficient to account for the large horizontal velocities encountered in the simulation. 

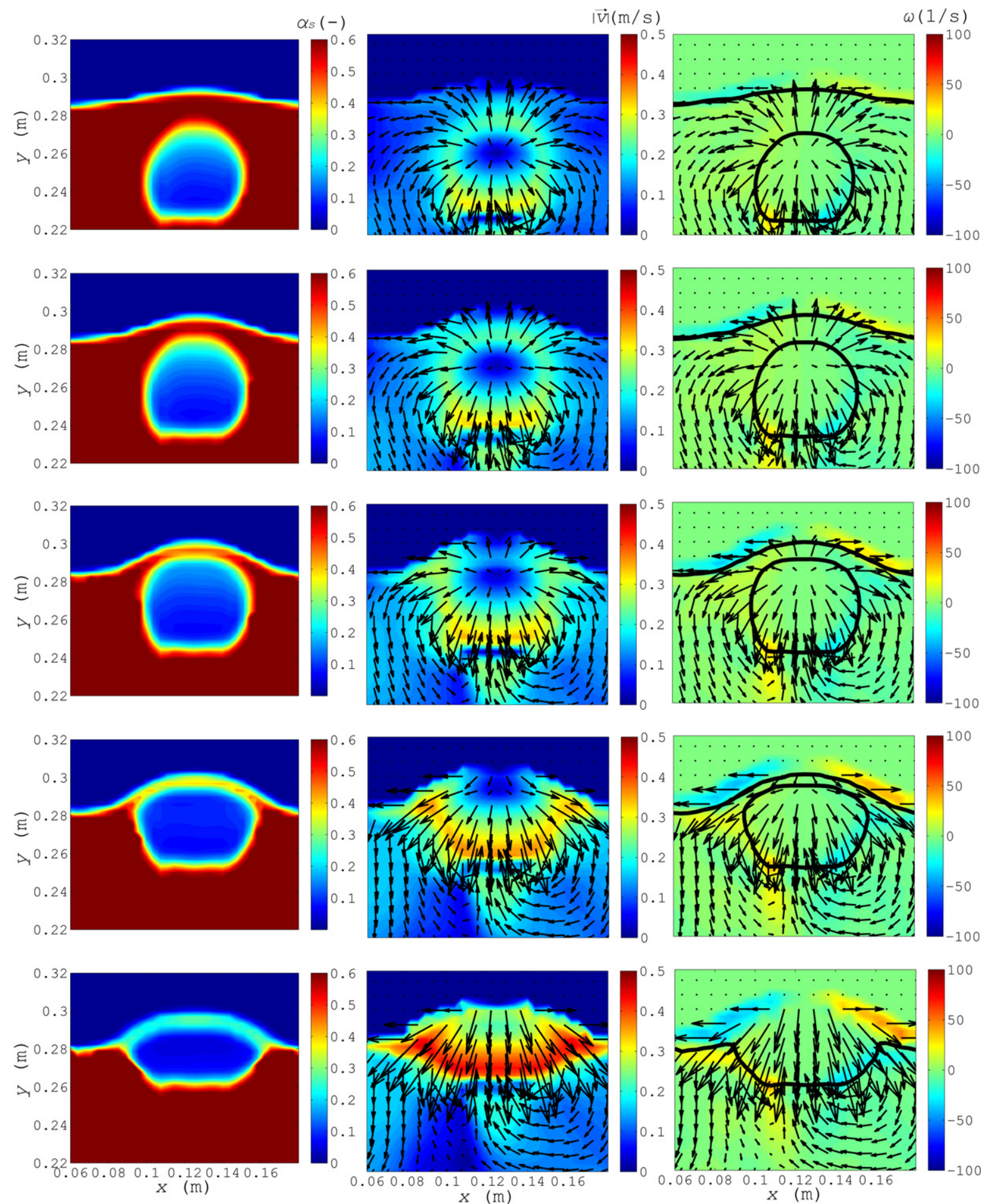

Fig. 7. Erupting bubble contours for case 1: rows correspond to different time instants, from $t=25 \mathrm{~ms}$ (upper row) to $t=75 \mathrm{~ms}$ (lower row), every $25 \mathrm{~ms}$, and columns correspond, from left to right, to solids volume fraction (-), solids velocity $(\mathrm{m} / \mathrm{s})$, and vorticity $(1 / \mathrm{s}) . U_{\mathrm{b}}=0.424 \mathrm{~m} / \mathrm{s}$ and $U_{\mathrm{g}}=5.9 \times 10^{-2} \mathrm{~m} / \mathrm{s}$.

For $t=25 \mathrm{~ms}$, Figs. 8 and 9 indicate that potential flow is no longer able to predict the velocity of the ejected particles. This can be explained by considering that for $t>0$, particles are ejected from the bubble with the result that the dome starts to deviate from the liquid-like behaviour, a requirement for the potential flow theory to hold. Furthermore, the bubble grows laterally more than vertically during bubble eruption, which reduces the particle velocity at the bubble dome compared to the predictions obtained from potential flow theory.
For simulation case 2 , an erupting bubble of equivalent diameter $D_{\text {eq }}=6.0 \times 10^{-2} \mathrm{~m}$, rise velocity $U_{\mathrm{b}}=0.484 \mathrm{~m} / \mathrm{s}$, and a velocity due to its growth of $U_{\mathrm{g}}=8.0 \times 10^{-2} \mathrm{~m} / \mathrm{s}$, has been selected. Fig. 10 shows contour maps of the solids volume fraction, solids velocity and vorticity of the solids for this bubble. Arrows indicating the velocity vectors have been superimposed onto the velocity and vorticity plots. For the sake of clarity, only the results for $t=0$ are shown. Similar to the isolated bubbles analysed in case 2 , the erupting bubble shown in Fig. 10 has a small satellite bubble, this time to the left 

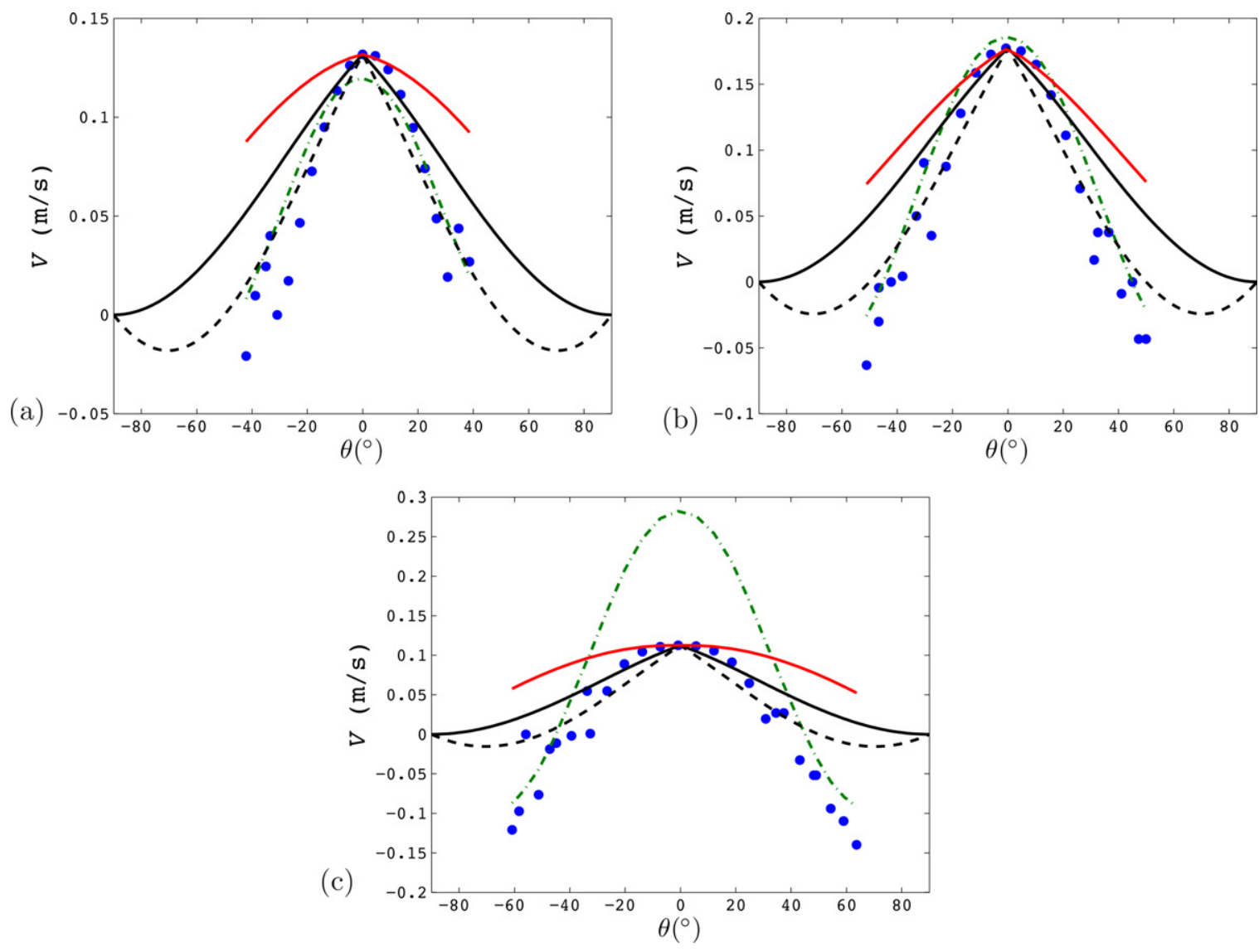

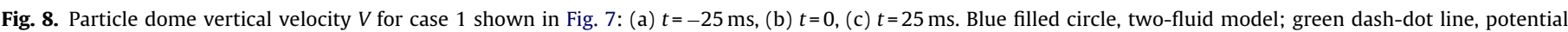

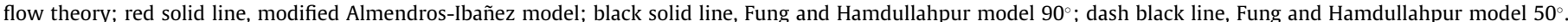
$U_{\mathrm{b}}=0.424 \mathrm{~m} / \mathrm{s}$ and $U_{\mathrm{g}}=5.9 \times 10^{-2} \mathrm{~m} / \mathrm{s}$. (For interpretation of the references to color in this figure legend, the reader is referred to the web version of the article.)

of the main bubble. Neither the velocity vectors nor the vorticity field appear to be perturbed by the small bubble except in a region very close to it. The largest values of the vorticity are encountered in the wake of the main bubble and in the region between the two bubbles.

In contrast to the erupting bubble analysed for case 1 , the bubble shown in Fig. 10 does not rise vertically, but at an angle of $\theta_{\mathrm{b}} \approx-13^{\circ}$ from the vertical direction. This small deviation of $\theta_{\mathrm{b}}$ from zero has an impact on the velocity profiles of the particles in the bubble dome, as illustrated in Fig. 11 which shows the vertical and horizontal velocities of the solids at time $t=0$. In particular, the two-fluid simulation results shown in Fig. 10 indicate that the vertical component of the particle velocity is larger on the left side of the dome than on the right side. It is thought that this asymmetry arises from the deformation of the bubble dome caused by the non-zero value of $\theta_{\mathrm{b}}$ [17].

Of the models considered here, potential flow theory gives the most accurate predictions for the vertical and horizontal velocity profiles for case 2 . This is in agreement with the findings for case 1 . At the sides of the bubble, i.e. $\theta<-20^{\circ}$ and $\theta>20^{\circ}$, there is excellent agreement between the vertical velocities predicted by the potential flow theory and the two-fluid simulation data in Fig. 11. The potential flow theory considered here accounts for a non-zero value of $\theta_{\mathrm{b}}$. However, the potential flow theory over-predicts the vertical velocities at the top of the bubble dome by approximately $15 \%$. The model from Almendros-Ibáñez et al. [17] also compensates for bubbles whose centroid does not rise in a perfectly vertical direction. This model gives an excellent prediction for the vertical velocities close to $\theta=\theta_{\mathrm{b}}$ but over-predicts the vertical velocity at large values of $\theta$.
The model of Fung and Hamdullahpur [16] does not account for the direction of bubble rise. As a result, the agreement between the vertical velocities predicted by this model and the simulation data is not only poor for large $\theta$, as was noted for case 1 , but also for $\theta=\theta_{\mathrm{b}}$. The distortion of the horizontal velocity profile due to the non-zero value of $\theta_{\mathrm{b}}$ is less pronounced than the deformation of the vertical velocity profile, as shown in Fig. 11. Therefore, as in case 1, the potential flow model provides the best predictions of $U$ at $t=0$.

The data presented in Fig. 11 indicate that the small bubble on the left of the bubble selected in case 2 does not have a noticeable impact on the profiles of the particle velocities in the bubble dome. Excluding the deformation induced by the non-zero value of $\theta_{\mathrm{b}}$, the velocity profiles for case 2 at $t=-25$ and $25 \mathrm{~ms}$ are similar to those shown in Figs. 8 and 9. For brevity, these data are not shown here.

The simulation parameters of case 2 where chosen to match the experiments reported by Müller et al. [18], who measured particle velocities in the domes of bubbles using PIV. In their study, Müller et al. [18] found that potential flow theory gave a better prediction for the velocity of particles in the dome of an erupting bubble than the correlation proposed by Fung and Hamdullahpur [16]. To allow a comparison to be made between the two-fluid simulations reported here and the experimental data reported by Müller et al. [18], a set of measurements of the vertical and horizontal particle velocity taken from [18] has been included in Fig. 11. These data were obtained for a bubble of equivalent diameter $D_{\text {eq }}=6.4 \times 10^{-2} \mathrm{~m}$ and velocity $U_{\mathrm{b}}=0.3 \mathrm{~m} / \mathrm{s}$, which are both similar to the erupting bubble studied for simulation case 2 . It should be noted that, compared to the current work, Müller et al. [18] used different definitions for the eruption angle, $\theta$, and for $t=0$. In that work, the data presented in Fig. 11 corresponds to $t^{\prime}=23 \mathrm{~ms}$, which 

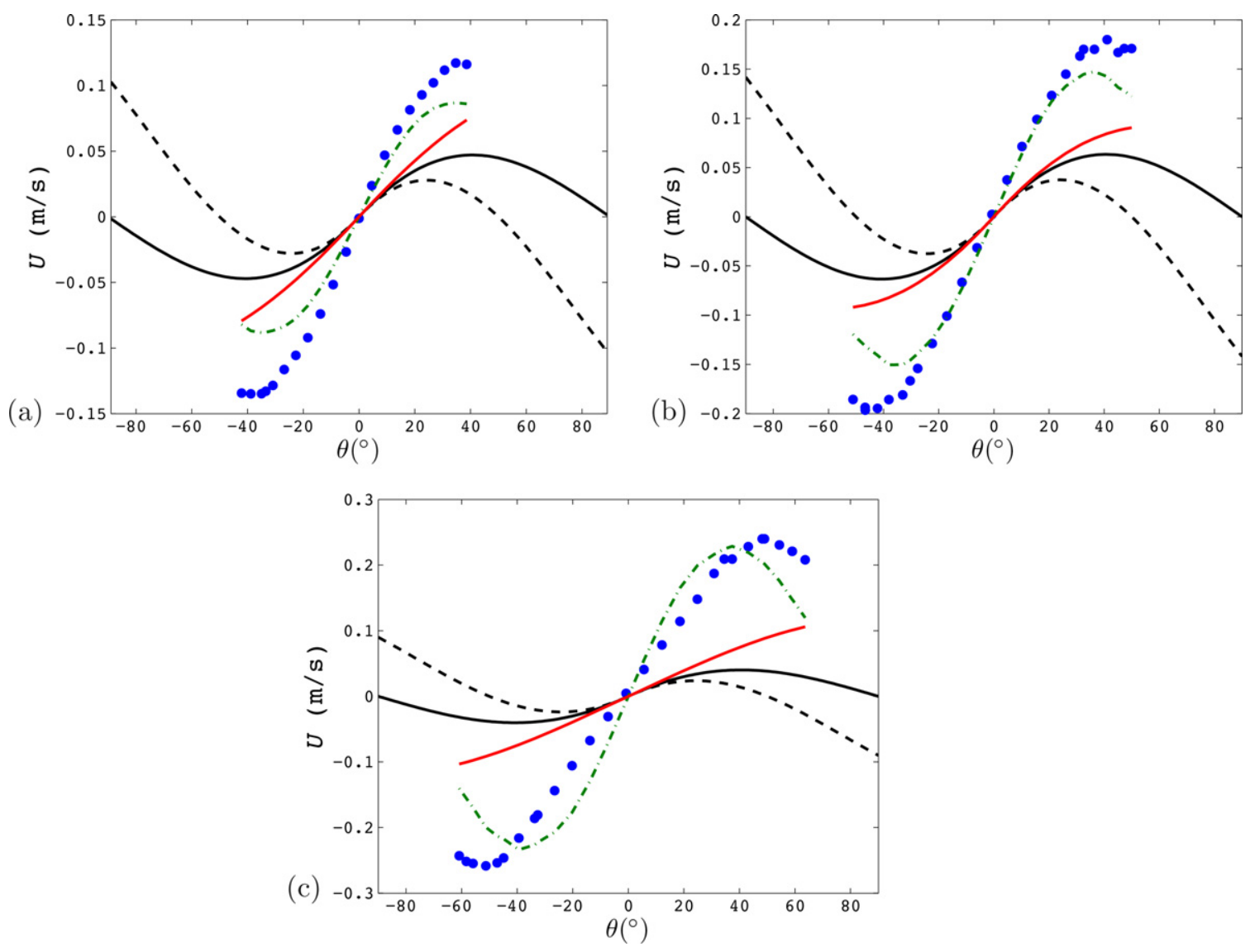

9. Particle dome horizontal velocity $U$ for case 1 shown in Fig. 7: (a) $t=-25 \mathrm{~ms}$, (b) $t=0$, (c) $t=25$ ms. Blue filled circle, two-fluid model; green dash-dot line, potential

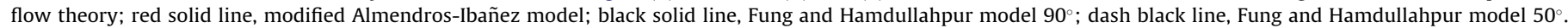
$U_{\mathrm{b}}=0.424 \mathrm{~m} / \mathrm{s}$ and $U_{\mathrm{g}}=5.9 \times 10^{-2} \mathrm{~m} / \mathrm{s}$. (For interpretation of the references to color in this figure legend, the reader is referred to the web version of the article.)

is equivalent to $t=0$ for the definition of time used here. Similarly, the data have been reprocessed employing the definition of $\theta$ used in this paper. Fig. 11 indicates that, although there is more scatter in the experimental data, there is good agreement between the experimental results reported by Müller et al. [18] and the two-fluid simulations reported here.

\subsection{Gas and particle flow direction in an erupting bubble}

The good agreement between the PIV results reported by Müller et al. [18] and the simulations reported here suggests that the twofluid simulation reproduces realistically the solids velocity during bubble eruption. Therefore, qualitative results from the two-fluid simulations regarding the gas velocities can be used with a certain level of confidence, although no direct validation with experimental data is possible due to the lack of published experimental data. Despite the fact that gas velocities have been measured in the freeboard close to erupting bubbles, e.g. [26-28], the existing experimental techniques do not allow a direct measurement of the gas velocity through the particles in the bubble dome. The lack of experimental measurements of the simultaneous particle and gas velocities in a two-dimensional erupting bubble means that, at present, such coupled information is only available from numerical "experiments".

Fig. 12 compares the angles that the simulated gas and solids velocities form, relative to the positive vertical direction. The angle, $\beta$, is given by:

$$
\beta=\arctan \left(\frac{U}{V}\right)
$$

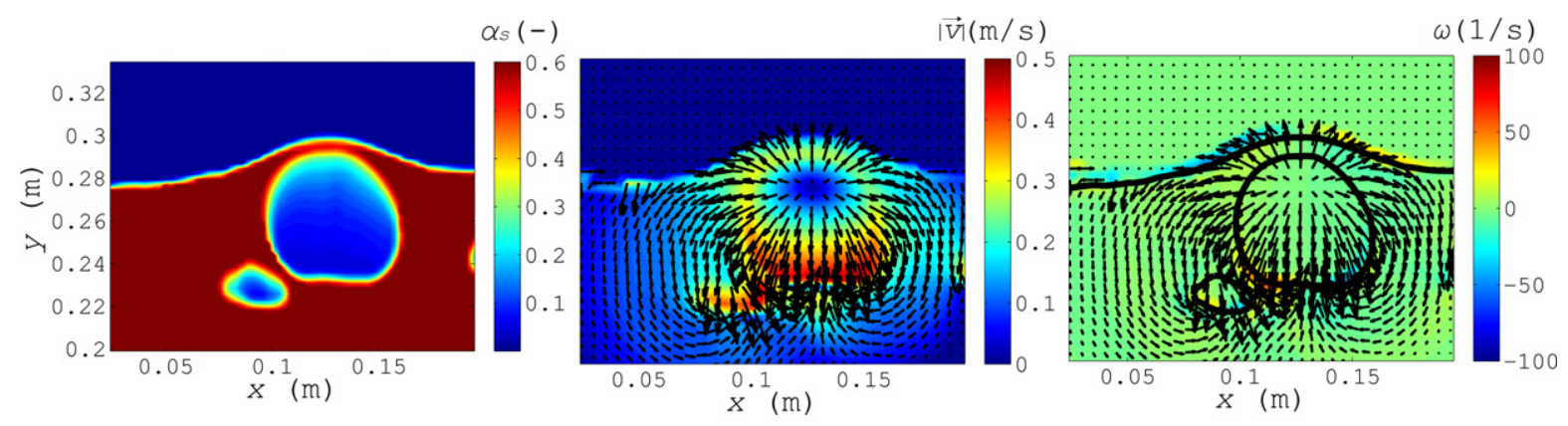

Fig. 10. Erupting bubble contours for case 2 : solids volume fraction $(-)$, solids velocity $(\mathrm{m} / \mathrm{s})$, and vorticity $(1 / \mathrm{s}) . U_{\mathrm{b}}=0.484 \mathrm{~m} / \mathrm{s}$ and $U_{\mathrm{g}}=8.0 \times 10^{-2} \mathrm{~m} / \mathrm{s}$. 

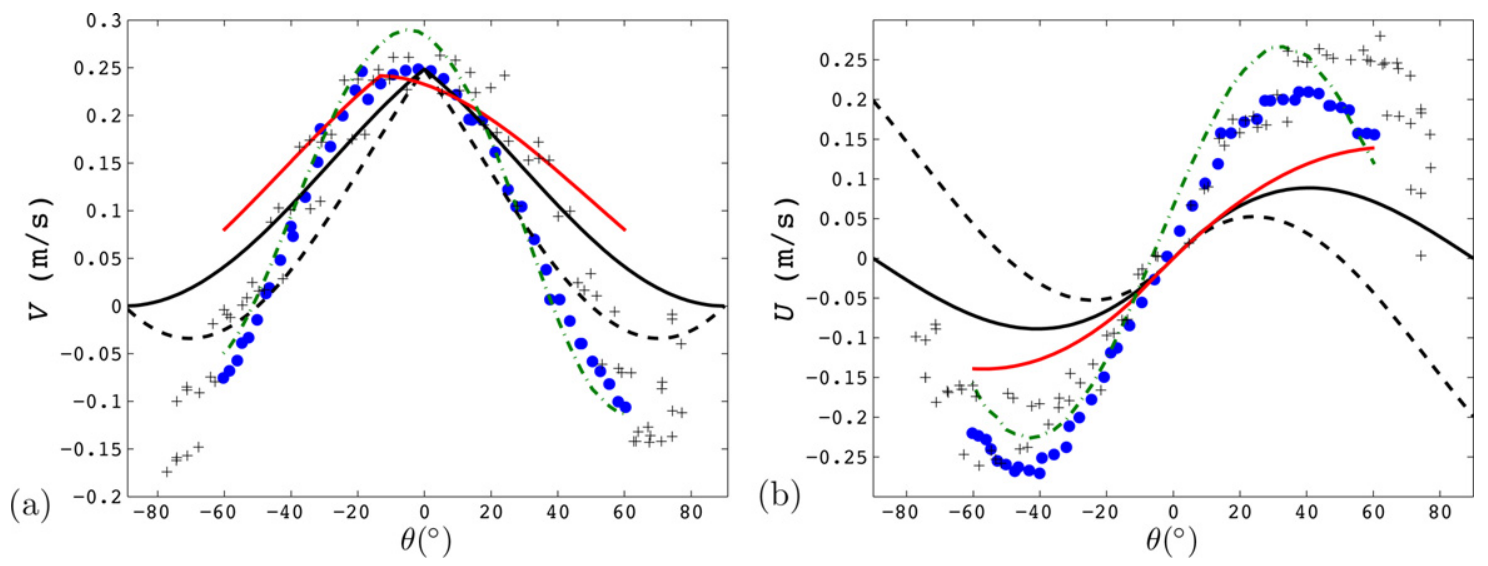

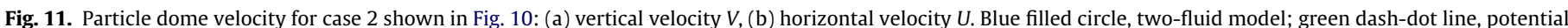

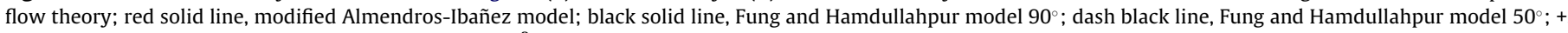

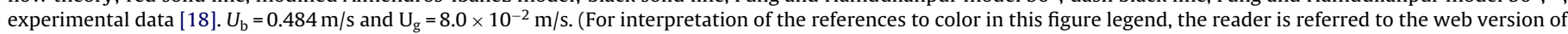
the article.)

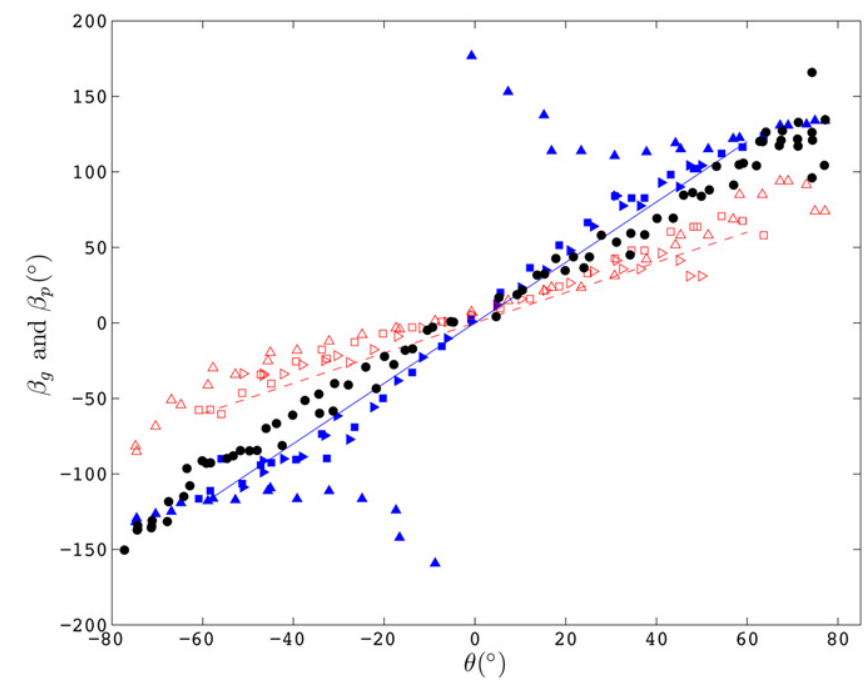

Fig. 12. Gas and particle velocity angles, $\beta_{\mathrm{g}}$ and $\beta_{\mathrm{p}}$, in the dome of an erupting bubble at several time instants for simulation case 1 . Also included the prediction $\beta_{\mathrm{p} \text {,pot }} \approx 2 \theta$ from the potential flow theory. Filled blue points, solids velocity; empty red symbols, gas velocity; blue solid line, $2 \theta$; red dash line, $\theta$; filled black circles, experimental data [18]. $\triangleright, t=0 \mathrm{~ms} ; \square, t=25 \mathrm{~ms} ; \Delta, t=50 \mathrm{~ms}$. (For interpretation of the references to color in this figure legend, the reader is referred to the web version of the article.)

In Fig. 12 , the angles formed by the gas, $\beta_{\mathrm{g}}$, and particle velocities, $\beta_{\mathrm{p}}$, are shown for times $t=0,25$ and $50 \mathrm{~ms}$. These data were obtained for simulation case 1 but analogous results can also be obtained using data from simulation case 2 . The results obtained from the experimental PIV data [18] plotted in Fig. 11 are also included here to verify the simulation results. Note that the results for $t=-25 \mathrm{~ms}$, that is, prior to particle ejection, are similar to those for $t=0$ and are not included for clarity.

It is clear from Fig. 12 that, except at the top of the bubble dome, the gas and particles in the dome of erupting bubbles move in different directions. In particular, the $\beta_{\mathrm{g}}$ points on Fig. 12 lie on a straight line of slope unity given by the equation $\beta_{\mathrm{g}}=\theta$. This indicates that the gas moves radially outwards form the centre of the erupting bubble, taking the shortest possible path out of the bubble. In contrast, the magnitude of the angle between the velocity of the particles and the vertical direction is always greater than would be expected for purely radial flow, i.e. $\beta_{\mathrm{p}}=\theta$.

It was demonstrated above that potential flow is able to predict the velocities of particles within the dome of an erupting bubble at $t=0$. Therefore, by substituting the velocities predicted by potential flow theory into Eq. (23), the following expression can be derived for the value of $\beta_{\mathrm{p}}$ predicted by potential flow theory:

$\beta_{\mathrm{p}, \mathrm{pot}}=\arctan \left(2 \frac{\tan (\theta)}{1-\tan ^{2}(\theta)}\right) \approx 2 \theta$

where $\theta=\arctan (x, y)$ The line $\beta_{\mathrm{p}}=2 \theta$ has been included on Fig. 12 and shows that the particle velocity angle $\beta_{\mathrm{p}}$ obtained from the two-fluid simulation is in good agreement with the angle estimated by potential flow theory around a cylinder. In addition, the particle velocity angle $\beta_{\mathrm{p}}$ has been calculated with Eq. (23) using the PIV data that was shown in Fig. 11. As the simulation data, the experimental results indicate that $\beta_{\mathrm{p}}$ is closer to $2 \theta$ than to $\theta$. This means that the particle velocity angle relative to the vertical direction is twice the radial direction angle, i.e. $\beta_{\mathrm{p}} \approx 2 \theta$. The success of potential flow theory in predicting $\beta_{\mathrm{p}}$ is more than remarkable taking into account that the approximation given by Eq. (24) holds even in the last instant before the bubble collapse, $t=25 \mathrm{~ms}$. For $t=50 \mathrm{~ms}$ the bubble has already collapsed and the potential theory around a cylinder is not able to reproduce the particle velocity direction, although the gas phase continues expanding radially.

\section{Conclusions}

The motion of particles around bubbles in 2D fluidized beds has been simulated using a two-fluid model. For isolated bubbles that are far from the freeboard, the simulated particle motion around bubbles is found to be described well by potential flow theory, a finding which is in agreement with experimental evidence [19]. For erupting bubbles good agreement is found between the simulated velocity profiles and the PIV data reported by Müller et al. [18]. Of the models for bubble eruption that have been tested here, the predictions of the potential flow theory shows the best agreement with results from PIV experiments and two-fluid simulations.

Both the simulation and experimental data indicate that particles are ejected from the dome of erupting bubbles at an angle of $2 \theta$ relative to the vertical direction, where $\theta$ is the angle that a radial line, connecting the bubble centroid to a position in the bubble dome, makes with the vertical. This observation is consistent with particle motion in the bubble dome being governed by potential flow theory, but is contrary to the standard models of a 2D bubble (e.g. [16] and related models), which assume that the particles are ejected radially outwards from the dome. 


\section{Acknowledgments}

This work has been partially funded by the Spanish Government (Project DPI2009-10518) and the Autonomous Community of Madrid (Project S2009/ENE-1660).

\section{References}

[1] D. Kunii, O. Levenspiel, Fluidization Engineering:, Butterworth-Heinemann, Newton, MA, 1991.

[2] D. Gidaspow, Multiphase Flow and Fluidization: Continuum and kinetic theory descriptions, Academic Press, San Diego, CA, 1994.

[3] Y. Tsuji, T. Kawaguchi, T. Yanaka, Discrete particle simulations of twodimensional fluidized-beds, Powder Technol. 77 (1993) 79-87.

[4] H.T. Do, J.R. Grace, R. Clift, Particle ejection and entrainment from fluidized beds, Powder Technol. 6 (1972) 195

[5] S.C. Saxena, A. Mathur, On the origin of solids projected from the surface of a gas-fluidized beds, Chem. Eng. Sci. 39 (1984) 917.

[6] S.E. George, J.R. Grace, Entrainment of particles from aggregative fluidized beds, AIChE Symp. Ser. 74 (1978) 67.

[7] S.T. Pemberton, J.F. Davidson, Elutriation from fluidized beds-I. Particle ejection from the dense phase into the freeboard, Chem. Eng. Sci. 41 (1986) 243.

[8] E.K. Levy, H.S. Caram, J.C. Dille, S. Edelstein, Mechanism for solid ejections from gas-solid fluidized beds, AIChE J. 29 (1983) 383.

[9] H. Hatano, M. Ishida, The entrainment of solids particles from a gas-solid fluidized bed, J. Chem. Eng. Jpn. 14 (1981) 306.

[10] J.R. Grace, F. Taghipour, Verification and validation of CFD models and dynamic similarity for fluidized beds, Powder Technol. 139 (2004) 99-110.

[11] G.N. Ahujaa, A.W. Patwardhan, CFD and experimental studies of solids hold-up distribution and circulation patterns in gas-solid fluidized beds, Chem. Eng. J. 143 (2008) 147-160.

[12] A. Busciglio, G. Vella, G. Micale, L. Rizzutia, Analysis of the bubbling behaviour of 2D gas solid fluidized beds: Part II. Comparison between experiments and numerical simulations via digital image analysis technique, Chem. Eng. J. 148 (2009) 145-163.

[13] N.G. Deen, M. van Sint Annaland, M.A. van der Hoef, J.A.M. Kuipers, Review of discrete particle modelling of fluidized beds, Chem. Eng. Sci. 62 (2007) 28-44

[14] B.G.M. van Wachem, A.E. Almstedt, Methods for multiphase computational fluid dynamics, Chem. Eng. J. 96 (2003) 81-98.
[15] D. Santana, S. Nauri, A. Acosta, N. Garcia, A. Macias-Machin, Initial particle velocity spatial distribution from 2-D erupting bubbles in fluidized bed, Powder Technol. 150 (2005) 1-8.

[16] A.S. Fung, F.A. Hamdullahpur, A gas and particle flow model in the freeboard of a fluidized bed based on bubble coalescence, Powder Technol. 74 (1994) 121.

[17] J.A. Almendros-Ibáñez, C. Sobrino, M. de Vega, D. Santana, A new model for ejected particle velocity from erupting bubbles in 2-D fluidized beds, Chem Eng. Sci 61 (2006) 5981-5990.

[18] C.R. Müller, J.F. Davidson, J.S. Dennis, A.N. Hayhurst, A study of the motion and eruption of a bubble at the surface of a two-dimensional fluidized bed using particle image velocimetry (PIV), Ind. Eng. Chem. Res 46 (2007) 1642-1652.

[19] D. Harrison, J.F. Davidson, Fluidised Particles, Cambridge University Press, Cambridge, 1963.

[20] M. Syamlal, W. Rogers, T.J. O’Brien, MFIX Documentation: Theory guide, U.S. department of Energy (DOE), Morgantown Energy Technology Center, Morgantown, West Virginia, 1993.

[21] S. Benyahia, M. Syamlal, T.J. O’Brien, Summary of MFIX equations 2005-4, 2007

[22] R.J. Hill, D.L. Koch, A.J.C. Lad, The first effects of fluid inertia on flows in ordered and random arrays of spheres, J. Fluid Mech. 448 (2001) 213-241.

[23] R.J. Hill, D.L. Koch, A.J.C. Ladd, Moderate Reynolds number flows in ordered and random arrays of spheres, J. Fluid Mech. 448 (2001) 243-278.

[24] S. Benyahia, M. Syamlal, T.J. O'Brien, Extension of Hill-Koch-Ladd drag correlation over all ranges of Reynolds number and solids volume fraction, Powder Technol. 162 (2006) 166-174.

[25] J.A. Almendros-Ibáñez, D. Pallarés, F. Johnsson, D. Santana, Novel approach to characterize fluidized bed dynamics combining particle image velocimetry and finite element method, Ind. Eng. Chem. Res. 48 (2009) 5010-5023.

[26] S. Vun, J. Naser, P.J. Witt, W. Yang, Measurements and numerical predictions of gas vortices formed by single bubble eruptions in the freeboard of a fluidised bed, Chem. Eng. Sci. 65 (2010) 5808-5820.

[27] G. Hartung, C.R. Müller, J. Hult, C.F. Kaminski, J.S. Dennis, Laser diagnostic investigation of the bubble eruption patterns in the free board of fluidized beds. Part 1: optimized Acetone PLIF measurements, Ind. Eng. Chem. Res. 47 (2008) 5686-5697.

[28] C.R. Müller, G. Hartung, J.F. Davidson, J. Hult, C.F. Kaminski, J.S. Dennis, Laser diagnostic investigation of the bubble eruption patterns in the free board of fluidized beds. Part 2: simultaneous acetone PLIF and stereoscopic PIV measurements, AIChE J. 55 (2009) 1369-1382. 